\title{
Diastolic Dysfunction Accompanies Alterations in Myocardial Structure, Cellular Composition and Macrophage Polarization in Survivors of lonizing Radiation Exposure
}

Kristofer T. Michalson, DVM, PhD, Gregory O. Dugan, DVM a , David L. Caudell, DVM, PhDª J. Mark Cline, DVM, PhD ${ }^{\mathrm{a}}$, Dalane W. Kitzman, $\mathrm{MD}^{\mathrm{b}}$, Thomas C. Register, $\mathrm{PhD}^{\mathrm{a} 1}$

\section{Departments of ${ }^{\mathrm{P}} \mathrm{Pathology} / \mathrm{Comparative}$ Medicine and ${ }^{\mathrm{b}}$ Internal Medicine-Cardiology, Wake Forest School of Medicine, Winston-Salem, NC, USA}

\section{Running title: Radiation Effects on the Heart}

${ }^{1}$ Address for correspondence: Department of Pathology, Section on Comparative Medicine, Wake Forest University School of Medicine, Medical Center Boulevard, Winston-Salem, NC 27157-1040; e-mail: register@wakehealth.edu.

Total Word Count: 7682

Subject Codes: Animal Models of Human Disease, Cardio-Oncology, Myocardial Biology, Pathophysiology, Translational Studies 


\begin{abstract}
Rationale: Radiation induced heart disease (RIHD) is a significant delayed/late effect of ionizing radiation exposure.
\end{abstract}

Objective: To determine the cardiac effects of total body irradiation (TBI) in male rhesus macaques, a translational non-human primate (NHP) model.

Methods and Results: Echocardiography was performed on survivors of a single dose (6.4-8.5 Gy) of TBI ( $n=34)$ and non-irradiated controls $(n=26)$ divided into longer (LT IRR) and shorter term (ST IRR) survivors and controls to assess the effects of time since TBI on phenotypes. LT IRR had increased Doppler transmitral early filling velocities $(E)$, decreased early mitral annular descent velocities (e'), and higher E/e' ratio compared to LT CTL (all $p \leq 0.05$ ), indicating left ventricular (LV) diastolic dysfunction. Echocardiographic stroke volume, cardiac output, and end-diastolic and systolic volumes were also lower in LT IRR than controls (all $p \leq 0.05$ ). ST IRR had similar alterations in LV diastolic function but not in cardiac volumetric measures. Analyses of LV, interventricular septum (IVS), and right ventricle (RV) myocardium from deceased irradiated animals $(n=17)$ exposed to a single dose (6.9-8.05 Gy) TBI and non-irradiated controls $(n=12)$ showed that IRR animals had decreased LV and IVS capillary density, and increased LV fibrosis, pan-cardiac fibroblast and macrophage staining, LV and IVS M2 macrophages, and pan-cardiac M1 macrophages (all $\mathrm{p}<0.05$ ). While M2 predominated over M1 macrophages in both groups, M1 showed greater increases than M2 in IRR.

Conclusions: LV diastolic dysfunction due to radiation exposure may be due to a combination of capillary rarefication, activation and expansion of fibroblasts, and increased accumulation of both pro-fibrotic and pro-inflammatory macrophages, all of which lead to increased myocardial stiffness due to fibrosis. Collectively, these findings provide insights into the delayed effects of 
acute ionizing radiation exposure and suggest that therapies targeting macrophage regulation of fibrosis may mitigate radiation induced heart disease.

\section{KEYWORDS}

macrophage polarization, extracellular matrix, myocardial inflammation, CD163, CD68, radiation risk; fibrosis; macrophage

\section{ABBREVIATIONS}

DEARE: $\quad$ Delayed effect of acute ionizing radiation exposure

RIHD: Radiation-induced heart disease

TBI: $\quad$ Total body Irradiation 


\section{INTRODUCTION}

Radiation-induced heart disease (RIHD) can be a delayed effect of acute ionizing radiation exposure (DEARE) of the heart, which can occur through accidental or malicious nuclear events, radiotherapies for lung or breast cancers and Hodgkin's disease, or during space flight. The condition is characterized by a broad spectrum of pathologies including pericarditis (primarily at total doses greater than $40 \mathrm{~Gy})^{1}$, arrhythmias, valvular disease, precocious coronary artery atherosclerosis, and myocardial fibrosis in humans. ${ }^{2-4}$ In humans, the delayed effects of acute radiation exposure (DEAREs) are perhaps best documented in the Life Span Study (LSS) of the Japanese survivors of the Hiroshima and Nagasaki atomic bombings, who generally received an estimated $\leq 2$ Gy total body irradiation. ${ }^{8}$ Studies of the LSS showed markedly increased predispositions for heart and cardiovascular disease ${ }^{9}$ as well as cancer $^{10}$ and noncancer diseases including stroke, ${ }^{11,12}$ chronic kidney disease, ${ }^{13,14}$ and respiratory disease ${ }^{15}$.

Despite recognition of RIHD as a significant clinical concern, there are no consensus recommendations on prevention or treatment of RIHD, due in large part to significant gaps in knowledge regarding the initiation and progression of local and systemic processes involved in RIHD, particularly due to lack of animal models truly relevant to humans. These gaps include the pathogenesis of myocardial remodeling, the effects of RIHD on systolic and diastolic function, whether these changes result in heart failure (HF) with reduced ejection fraction (HFrEF) or heart failure with preserved ejection fraction (HFpEF), and how the condition progresses over time.

Myocardial fibrosis is a key pathologic phenotype of RIHD leading to adverse myocardial remodeling, but the mechanisms underlying its development, particularly the critical events involved and pertinent signaling pathways which might be targeted therapeutically are not fully understood, and initiating signals and cells promoting fibrosis are largely unknown. Our laboratory recently demonstrated that the key protein responsible for monocyte chemotaxis 
(MCP1) was elevated $(\mathrm{p}<0.05)$ in previously irradiated male rhesus macaques years after irradiation (5.6-9.7 years), suggesting a long term pro-inflammatory systemic environment in which monocyte/macrophages may play a role in the pathogenesis of RIHD fibrosis. ${ }^{23}$ We also recently discovered there are alterations in circulating monocyte polarization from classical towards intermediate monocyte phenotypes after 6 months after total body irradiation, providing a potential mechanism for RIHD fibrosis in the heart and other organs ${ }^{25}$.

The goal of this study was to determine the effects of TBI on 1) the structure and function of the heart using echocardiography and 2) the cellular and extracellular matrix composition of the myocardium using immunohistochemistry and histology to determine alterations which might play a role in RIHD. This study utilized a unique resource of male rhesus macaque survivors, termed the Radiation Survivor Cohort (RSC), that were previously exposed to single dose total body irradiation (TBI). ${ }^{23,30-32}$ For functional outcomes, we specifically focused on two subsets of the RSC which differed in time elapsed since irradiation to determine the potential for long term progression which might lead to heart failure.

\section{METHODS}

\section{Animals}

Subjects were male rhesus nonhuman primates (NHP; Macaca mulatta) obtained from the Armed Forces Radiobiology Research Institute (AFRRI), the University of Maryland (UMD), the University of Illinois at Chicago (UIC), the Lovelace Respiratory Research Institute, and the Michale E. Keeling Center for Comparative Medicine and Research. Animals were screened by intradermal tuberculin testing three times a year to exclude tuberculosis, and by immunoassay upon arrival for simian retroviruses (Washington National Primate Research Center, Seattle, WA). Animals were aged based on dentition, weighed, examined by a veterinarian and quarantined for 60 days upon arrival per standard institutional protocol. The animals were assessed and treated for discomfort and illness as described previously by $\mathrm{DeBo}^{23}$ including criteria for humane euthanasia. All experimental procedures were conducted in compliance with 
the Wake Forest School of Medicine (WFSM) Institutional Animal Care and Use Committee requirements and followed the Guide for Care and Use of Laboratory Animals.

In vivo: Echocardiographic phenotyping

Two groups of animals were chosen from our available dataset of $60 \mathrm{NHP}$ for the current study of echocardiographic phenotypes, 1) an older, longer term survivor group of 14 mature adult (12.6 to 20.3 year old) long-term survivors ( $L T$ IRR) exposed to a single TBI dose of 6.5 to 8.4 Gy approximately 9.7 to 11.6 years earlier, which were compared to 15 age-matched, nonirradiated controls (LT CTL)(Table 1), and younger, shorter term survivor group (ST IRR) of 20 young adult (4.7 to 6.0 year old) exposed to a single TBI dose of 6.4-8.5 Gy approximately 2.16 to 2.96 years earlier, which were compared to 10 age-matched, nonirradiated controls (ST CTL)(Table 1).

Ex vivo: Histochemical and immunohistochemical phenotyping of the heart

Cardiac tissues were obtained from a separate set of animals that had died or been euthanized due to morbidity. All subjects underwent a complete necropsy with saline flush as detailed in DeBo et al. ${ }^{23}$ Subjects included 17 irradiated animals (Deceased IRR) exposed to a single TBI dose of 6.92 to 8.05 Gy TBI approximately 2.1 to 10.6 years prior that died or were euthanized at 5.6 to 19.2 years of age (mean 12.1 years), along with 12 non-irradiated controls (Deceased CTL) that died or were euthanized at 6.8 to 17.3 years of age (mean 9.3 years)(Table 1). Longitudinal sections of LV, interventricular septum (IVS) and right ventricle (RV) were sectioned from paraformaldehyde fixed hearts as previously described. ${ }^{40}$

Diet

Prior to arrival at WFSM (and during the time of irradiation), animals consumed standard laboratory primate chow diet (18\% protein, $13 \%$ fat and $69 \%$ carbohydrates, with a low cholesterol content of 0.023 mg/Cal (for example, Monkey Diet 5038; LabDiet®, St. Louis, MO). After arrival at WFSOM, monkeys were transitioned to a moderately atherogenic Western diet developed by WFSM and Purina® (Typical American Diet, TAD; Purina LabDiet as NHP Diet) 
which contained $18.4 \%$ protein, $36.2 \%$ fat and $45.4 \%$ carbohydrates as percentage of calories provided, and a cholesterol content of $0.18 \mathrm{mg} /$ Cal. Any animals with a hemoglobin A1c higher than $6.5 \%$, indicating diabetes mellitus onset, were transitioned to standard laboratory chow in the interest of health preservation.

\section{In Vivo: Echocardiography}

Animals were sedated with $10-15 \mathrm{mg} / \mathrm{kg}$ ketamine and 0.05-0.15 mg/kg midazolam to facilitate echocardiography using a Logiq S8 ultrasound system (GE Healthcare, Chicago, ILL) by trained NHP ultrasonographers following guidelines for image acquisition and analysis established by the American Society of Echocardiography and European Association of Cardiovascular Imaging ${ }^{41,42}$ combined with recommendations specifically for rhesus macaque echocardiograms reported by Korcarz et al. ${ }^{43}$ Captured images and video recordings were subsequently analyzed using Image-Arena software (TomTec Imaging Systems GMBH, Munich, Germany). Heart rate and blood pressure was obtained throughout the echocardiogram using electrocardiography and blood pressure cuff respectively. Parasternal short and long axis, and apical two (A2C) and four chamber (A4C) views of the heart were obtained to evaluate structure and systolic and diastolic function. Parasternal short axis brightness (B) mode views at the level of the aortic valve were used to measure end systolic aortic root and left atrial diameters to calculate the aortic root ( $A O)$ to left atrium (LA) diameter ratio (AO/LA). Motion (M) mode views at the level of the LV papillary muscles were captured to assess interventricular septal thicknesses at end diastole (IVSd) and end systole (IVSs), LV internal diameters at end diastole (LVIDd) and end systole (LVIDs), LV posterior wall thicknesses at end diastole (LVPWd) and end systole (LVPWs), and fractional shortening (FS). The LV endocardium was traced at end diastole and end systole in $\mathrm{A} 2 \mathrm{C}$ and $\mathrm{A} 4 \mathrm{C}$ views to obtain end diastolic (EDV) and systolic volumes (ESV) by method of disks, stroke volume (SV), and cardiac output (CO), that were combined to obtain biplane EDV, ESV, SV and CO values. The LA endocardium was traced at end systole in $A 2 C$ and $A 4 C$ views to calculate BP LA end systolic volume (LA Vol BP). Pulsed 
wave Doppler images taken at the mitral valve inflow tract in the $\mathrm{A} 4 \mathrm{C}$ view were used to measure early $(E)$ wave and late $(A)$ wave peak filling velocities, $E$ wave deceleration slope, $E$ wave deceleration time, and the E/A ratio. Tissue Doppler imaging of the lateral annulus of the mitral valve in the A4C view was used to assess early (e') and late (a') mitral annular descent velocities, derive the e'/a' ratio and complete the calculation for the E/e' ratio, an index of LV filling pressure ${ }^{44}$. Pulsed wave Doppler images taken at the aortic outflow tract were used to measure aortic valve mean ( $\mathrm{V} \vee \mathrm{Vmean}), \max (\mathrm{AV} \vee \max$ ) and velocity time integral (AV VTI). The LV outflow tract diameter (LVOT) at beginning systole was measured using the parasternal long axis view. Body surface area $\left(B S A, \mathrm{~m}^{2}\right)$ at the time of echocardiogram was calculated as $\mathrm{BSA}=$ Body weight $^{2 / 3} \times 0.0969$, utilizing the animal's body weight $(\mathrm{kg})^{45}$. In some animals, complete echocardiograms were not obtained due to difficulties in complete visualization of cardiac structures.

\section{Ex vivo: Immunostaining and Image Capture}

Paraformaldehyde fixed, paraffin-embedded sections of longitudinally oriented LV $(n=29)$, IVS ( $n=26)$, and RV $(n=26)$ were serially sectioned and processed for histologic and immunohistochemistry $(\mathrm{IHC})$. One section from each area was stained with Masson's trichrome to assess fibrosis extent. Appropriate monoclonal antibodies were identified and protocols and procedures were developed and optimized for IHC staining of the phenotypes of interest. Subsequent serial sections were immunostained using a Leica Bond RX autostainer (Leica Biosystems, Inc, Buffalo Grove, IL) for CD31 ( $L V n=26$, IVS n= 24, RV n=22) to assess endothelium/capillary density, (1:50, clone JC70A, Thermo Scientific, Waltham, MA), S100A4 ( $L V n=25$, IVS $n=26, R V n=22$ ) for fibroblasts and cells undergoing epithelial to mesenchymal transition (1:1000, clone CPR2761(2), Abcam, Cambridge, MA), CD68 (LV n=25, IVS n= 26, RV $\mathrm{n}=22$ ) for macrophages (1:800, clone KP1, Dako/Agilent, Santa Clara, CA), and CD163 (LV $n=26$, IVS $n=25, R V n=22)$ for macrophages (1:800, clone EDHu-1, Thermo Scientific, Waltham, MA), all detected using the Vector Red alkaline phosphatase substrate detection kit 
(Vector Laboratories, Inc, Burlingame, CA). Finally, sections were dual stained with CD163 (Vector Red) and either pSTAT1 (DAB)(1:25, clone 58D6, Cell Signaling Technologies, Danvers, MA) or c-Maf (DAB)(1:50, clone EPR16484, Abcam, Cambridge, MA) to assess M1 or M2 macrophages as previously described. ${ }^{46}$ All slides were digitally scanned using a $20 \mathrm{X}$ objective on a BX61VS microscope and acquired on a VS 120 virtual slide imaging system (Olympus Corporation, Tokyo, Japan).

\section{Tissue Section Image Analysis}

Acquired images were assessed using semi-automated algorithms developed internally specifically for phenotypes of interest on the Visiopharm Integrator System (Visiopharm, Broomfield, $\mathrm{CO}$ ). First, a region of interest (ROI) was manually outlined around the myocardium, excluding valvular insertion points, the epicardium, papillary muscles and superficial large coronary arteries. The ROI was subsequently assessed via an automated algorithm that determined tissue area from non-tissue area (e.g. space around tissue or induced by artifactual shrinkage) using a green pixel filter thresholded to the background for each slide.

The ROI was then quantitated by custom-designed algorithms specific to the stain or antibody of interest. For trichrome stains, the blue stained area representing fibrosis was quantified after defining the range of blue pixel values and was subsequently divided by the total tissue area previously obtained to calculate percent fibrotic area. For IHC stains for CD31, S100A4, CD68, and CD163, the positive area of interest was determined via thresholding using a red-green pixel contrast filter and divided by the total tissue area to calculate positive percentage area for CD31 or a normalized area (corrected for tissue area) for S100A4, CD68, and CD163. Additional steps to quantitate dual stained pSTAT1/CD163 and c-Maf/CD163 sections included quantifying the total CD163 positive area as described above, then running an algorithm that outlined CD163 stained areas to create new ROls. The DAB positive area within these ROls was thresholded using the DAB specific filter developed by Visiopharm. ROls containing positive $D A B$ staining were subsequently converted to a dual stain positive area and quantified. 
Finally, dual stained pSTAT1/CD163 and c-Maf/CD163 positive areas were normalized to the mean tissue area.

Statistics

All analyses were performed using Statistica ver. 13.3 (TIBCO Software, Palo Alto, CA). All data were analyzed for normality and found to be normally distributed. Age, body weight, and BSA phenotypes were analyzed using Student $t$ tests. Echocardiographic phenotypes were analyzed via analysis of co-variance (ANCOVA) and least square differences (LSD) post-hoc tests using BSA as a covariate. Histochemical and immunohistochemical outcomes were also covaried for BSA at time of death via ANCOVA and LSD post-hoc tests. Statistics are presented based on BSA corrected values, means \pm SEM of the raw data not corrected for BSA are presented in the tables. The level of significance was set at $p \leq 0.05$.

\section{RESULTS}

\section{Echocardiographic Phenotypes of Long Term Survivors}

Structural and functional differences between LT IRR and LT CTL animals were assessed using B mode, M mode, and pulsed wave and tissue Doppler echocardiography (Supplementary Table 1). LT IRR animals differed from LT CTL in several diastolic function measures including increased transmitral early filling velocities $(E)$ and $E$ wave deceleration slope and decreased early mitral annular descent velocities (e') that resulted in higher E/e' ratios and decreased early (e') to late (a') mitral annular descent velocity ratios (e'/a') (all $p \leq 0.05)$ (Figure 1). The remaining diastolic function measures of transmitral late filling velocity $(A), a^{\prime}$, and $E / A$ ratio were not different between groups. Stroke volume (biplane and apical 4 chamber views), cardiac output (apical 4 chamber view), and end diastolic volume (apical 4 chamber views), were lower in LT IRR than LT CTLs (all $p \leq 0.05$ ) and biplane measures of cardiac output and end diastolic volume tended to be lower as well (both $p=0.07$ ) (Figure 2). M-mode measures of cardiac structure corroborated our previous findings of decreased left ventricular diameters at end 
systole (LVIDs) and diastole (LVIDd) in LT IRR animals compared to LT CTLs, and also showed trends for decreased LV posterior wall thickness at end systole (LVPWs) $(p=0.07)$ and diastole (LVPWd) ( $p=0.06)$ (Supplementary Table S1). No differences were observed in interventricular septal thicknesses at end systole (IVSs) or end diastole (IVSd) (both p> 0.5). Parasternal long axis measurement of the LV outflow tract diameter (LVOT) showed that LT IRR animals had significantly narrower outflow tract diameters compared to LT CTL $(p=0.01)$ (Supplementary Table S1). No differences in heart rate, blood pressures (systolic, diastolic, and mean), ejection fraction, fractional shortening, or left atrium volume and size measures; aortic root to left atrium ratio (AO/LA) and left atrial end systolic volume (biplane) (LA Vol BP) were found.

\section{Echocardiographic Phenotypes of Short Term Survivors}

Structural and functional differences between ST IRR and ST CTL animals were evaluated similarly as LT IRR vs LT CTLs (Supplementary Table S2). Like LT IRR animals, ST IRR animals also had increased E/e' ratios and decreased e' and e'/a' ratios compared to ST CTLs (all $p \leq 0.05$ ) (Figure 3), while $E, A, E / A$ ratio, and a' were not significantly different between groups. ST IRR and ST CTL did not differ in cardiac output, stroke volume, end diastolic volumes, heart rate, blood pressures (systolic diastolic, and mean), ejection fraction, fractional shortening, IVSd, ESV, LVIDs, LVIDd, AO/LA, or LA Vol BP. Interestingly, ST IRR animals had significantly thickened LVPWs, LVPWd, and IVSs (all p $\leq 0.05$ ) compared to ST CTL (Supplementary Figure 1).

Histological and Immunohistochemical Staining of the Left Ventricular, Interventricular Septal, and Right Ventricular Myocardium

Sections of LV, IVS, and RV from 17 deceased previously irradiated animals (Deceased IRR) and 12 deceased controls (Deceased CTL) were evaluated for structural and cellular components using histochemical and immunohistochemical stains for fibrosis (Masson's trichrome), capillary density (CD31), fibroblasts (S100A4), macrophages (CD68 and CD163) and M1 or M2 macrophages (pSTAT1/CD163 and c-Maf/CD163 respectively). IRR animals had 
significantly greater positive trichrome staining of the LV $(p<0.05)$, and no significant difference in the RV or IVS compared to CTL animals. (Figure 4) Capillary density was reduced in the IRR group in the LV $(p<0.05)$ and IVS $(p<0.01)$, but not the RV. (Figure 4) IRR myocardium exhibited increased S100A4 $4^{+}$area in all cardiac sections (LV $p<0.01$, IVS $p<0.001$, and RV $p<0.05$ ) compared to CTL (Figure 5). In addition, approximately 100 fold less CD68 ${ }^{+}$macrophage staining area $\left(\mathrm{LV}\right.$ mean $=8900 \mathrm{um}^{2}$, IVS mean $\left.=6134 \mathrm{um}^{2}, \mathrm{RV}=3484 \mathrm{um}^{2}\right)$ was observed relative to $\mathrm{CD}_{163^{+}}$macrophage staining area $\left(\mathrm{LV}\right.$ mean $=905232 \mathrm{um}^{2}$, IVS mean $=720474 \mathrm{um}^{2}$, $\mathrm{RV}$ mean $=339053 \mathrm{um}^{2}$ ) across all animals, and CD163 staining frequently co-localized with CD68 staining (Figure 6). Based on these findings we subsequently used CD163 as a panmacrophage marker and used nuclear staining PSTAT1 or c-Maf to indicate M1 or M2 like macrophages respectively. Deceased IRR animals had increased CD163 ${ }^{+}$macrophage staining in the LV $(p<0.05)$, IVS $(p<0.01)$, and RV $(p<0.05)$, increased $c-\mathrm{Maf}^{+} / \mathrm{CD} 163^{+}$ macrophages in the LV $(p<0.05)$ and IVS $(p<0.01)$, but not $R V$, and increased $p S T A T 1^{+} / C D 163^{+}$ macrophages in the LV $(p<0.01)$, IVS $(p<0.01)$, and RV $(p<0.05)$.(Figure 8) Finally, we compared the $\mathrm{c}-\mathrm{Maf}^{+} / \mathrm{CD} 163^{+}(\mathrm{M} 2)$ to $\mathrm{pSTAT1}{ }^{+} / \mathrm{CD} 163^{+}(\mathrm{M} 1)$ ratio and found that IRR animals had a significant decrease in the M2/M1 ratio across all assessed regions of the heart (all $\mathrm{p}<0.05)$.(Figure 7)

\section{DISCUSSION}

The present study demonstrates delayed effects of acute ionizing radiation exposures on the heart including 1) diastolic dysfunction that was worse in long term than short term survivors, 2) decreased cardiac volumetric measures in long term survivors, 3) increased left ventricular fibrosis, 4) decreased left ventricular and interventricular septal capillary density, and 5) increased fibroblast, pan-macrophage, M1 macrophage, and M2 macrophage staining in all cardiac regions, with a greater relative increase in M1 macrophages. Our most consistent finding was left ventricular diastolic dysfunction (LVDD), which was present in both younger (4.7 to 6.0 years old) short-term survivors (ST IRR) (2.16 to 2.96 years post-irradiation) and 
worsened in older (12.6 to 20.3 years old) long-term survivors (LT IRR) (9.7 to 11.6 years postirradiation) compared to age matched controls for each group. Both ST IRR and LT IRR groups had decreased early mitral annular descent velocities (e'), one of the earliest and consistent echocardiographically derived indicators of LVDD. ${ }^{47}$ This measure of LV relaxation decreases with increased myocardial stiffness and is generally not influenced by preload, unlike transmitral early filling peak velocity $(E)^{48}$ which was elevated in LT IRR relative to LT CTL but did not differ between ST IRR and ST CTL. E can be corrected for the influence of preload and relaxation by dividing it by e', and the resulting E/e' ratio has been demonstrated to be the best index to detect LVDD (86\%) in heart failure with preserved ejection fraction (HFpEF) in human patients who also had LVDD diagnosed via invasive conductance catheter derived pressure-volume loop $(\mathrm{PVL})$ analyses. ${ }^{49}$

Our data suggests that diastolic function may decline fairly soon after TBI exposure, which has also been noted in human breast cancer patients receiving contemporary radiotherapy ${ }^{57}$, and that effects on cardiac volumetric measures may require more time to develop as we only observed differences in the LT IRR vs. LT CTLs.

In addition to differences in diastolic function, we also found significant differences in echocardiographically determined cardiac structure. LT IRR animals had decreased LV internal diameters (LVIDd and LVIDs) compared to LT CTL, which corroborates our previous work in the RSC that used $13 / 20$ of the LT IRR and 11/11 LT CTL animals reported here. ${ }^{23}$ In contrast, we did not detect differences in LV internal diameters in ST IRR compared to ST CTLs, which were younger and more recently irradiated, although there were significant differences between ST IRR and ST CTL in thickness of the LV posterior wall at end systole and diastole and the IVS at end diastole. Interestingly, these cardiac dimensions are nearly identical in LT IRR and ST IRR animals, whereas ST CTL animals have numerically smaller LV posterior wall and IVS thicknesses compared to LT CTLs, accounting for the findings of significance. This difference 
may be accounted for by a relatively young age of the ST cohort monkeys, as male rhesus macaques do not plateau in body size until around 10 years of age..$^{58}$

Among deceased subjects, LV fibrosis extent was significantly increased in IRR compared to CTL animals and tended to be increased in the IVS, but not in the RV. However, the RV also had the highest percentage of fibrosis in both comparison groups, perhaps a function of the thinner RV wall compared to LV and IVS. The difference in fibrosis extent by myocardial region may also be related to reduction in capillary density, which has been demonstrated as a late effect in the LV of mice ${ }^{5,6}$, rats ${ }^{6}$, and rabbits ${ }^{7}$. Similar to the findings in these models, we observed a decrease in CD31 staining in the LV, and additionally the IVS, but not the RV.

Cardiac fibroblast content assessed using S100A4 (aka fibroblast specific protein 1, FSP1) was significantly elevated in all regions of TBI myocardium at a level of $10 \mathrm{x}$ that of $\mathrm{CD} 8^{+}$, the most commonly referenced macrophage marker colocalizing with $\mathrm{S} 100 \mathrm{~A} 4{ }^{60,63}$, indicating that the majority of S100A4 staining was due to cardiac fibroblast expansion.

Macrophage markers CD68 and CD163 were both elevated in myocardium from TBI subjects. CD163 appears to be a better general macrophage marker than $\operatorname{CD}^{4} 8^{46}$, so we used CD163 as a pan-macrophage marker and used dual staining and co-localization IHC with pSTAT1, a transcription factor elicited by interferon signaling ${ }^{66}$, to specifically identify proinflammatory M1 polarized macrophages, or c-Maf, a transcription factor necessary for IL10 production $^{67}$, to identify anti-inflammatory/reparative M2 polarized macrophages ${ }^{46}$. In both controls and TBI subjects, M2 staining phenotypes predominated over M1. TBI led to increased macrophage infiltration of the heart, with both $M 1$ and $M 2$ populations increased in comparable numbers, and a greater proportional increase in M1 macrophages due to a relatively sparse population of M1 macrophages in CTL myocardium. ${ }^{46}$ Collectively, this suggests that years after the initial IR exposure there is a long lived pro-inflammatory stimulus (M1) and repair (M2) by macrophage populations which leads to an accumulation of extracellular matrix and fibrosis. 
Our studies are consistent with long term consequences of ionizing radiation on diastolic dysfunction recently tied to an increased risk of $\mathrm{HF}$, specifically $\mathrm{HFpEF},{ }^{56}$ where women who had received contemporary radiotherapy for breast cancer (overall mean cardiac radiation dose of $3.3 \pm 2.7$ Gy, mean interval from radiotherapy to HF of $5.8 \pm 3.4$ years) had significantly increased odds ratios (OR) for HF (OR=9.14) and HFpEF (OR=16.9), but not HFrEF, even after adjustment for age, history of ischemic heart disease, history of atrial fibrillation, and cancer stage. $^{56}$

Macrophages are master regulators of inflammation and fibrosis, as they play key roles in the initiation, production, and resolution of fibrosis. ${ }^{64}$ In the case of myocardial infarction, proinflammatory (M1) and anti-inflammatory/reparative monocyte/macrophages (M2) sequentially infiltrate murine hearts after infarction, M1 macrophages dominate the early phase before giving way to increasing M2 macrophages and extracellular matrix deposition. ${ }^{65}$ Depletion of circulating monocytes during the early stages after infarction impaired healing and increased necrotic debris accumulation, depletion during the later stages decreased collagen deposition and endothelial cells. ${ }^{65}$ We previously demonstrated that total body irradiation depletes circulating monocytes over the first month following irradiation which could lead to a similar outcome, with accumulated damage leading to a long term dysregulated inflammation and repair cycle which leads to fibrosis. Further studies are needed to explore this concept.

There are advantages and limitations inherent in our study design. Our nonhuman primate echocardiographic image acquisition and analyses were done in accordance with human echocardiography standards with minimal adaptation for rhesus macaques, allowing easy translatability of our findings. For the tissue analyses, quantification of immunohistochemical stains were unbiased, being tightly controlled based on background pixel values of acquired digital images and performed on the entire myocardium in each region. It is also worth noting that the non-irradiated control hearts available for histochemical and IHC analyses were derived largely from monkeys that were in declining health which led to their 
necropsy. In addition, macrophage polarization is more of a spectrum than a dichotomy, so our dual labeled immunohistochemical approach to identify "M1" and "M2" cannot fully characterize the macrophage phenotypes in the myocardium. Future studies using state of the art single nucleus RNAseq and other techniques will help to explore in depth the characteristics of the macrophages in the myocardium.

In summary, irradiated animals showed 1) diastolic dysfunction that was worse in long term than short term survivors 2), decreased cardiac volumetric measures in long term survivors 3), increased left ventricular fibrosis 4), decreased left ventricular and interventricular septal capillary density 5), increased fibroblast, pan-macrophage, M1 macrophage, and M2 macrophage staining in all cardiac regions, with a greater relative increase in M1 macrophages. These findings provide insight into the delayed effects of acute ionizing radiation exposure on the heart and indicate that novel therapies targeting the initiation, regulation, and resolution of myocardial inflammation and fibrosis by macrophages have potential to mitigate radiation induced heart disease and perhaps fibrosis in other tissues.

\section{ACKNOWLEDGMENTS}

We acknowledge the support of the Comparative Pathology Laboratory Shared Resources of the Comprehensive Cancer Center of Wake Forest Baptist Medical Center. We thank Jean Gardin, Cathy Mathis, Renae Hall, Russell O'Donnell, Chrystal Bragg, Matt Dwyer, Michael Bennett, J. D. Bottoms, and Kathy Stewart for their technical assistance.

\section{SOURCES OF FUNDING}

This study was supported by Department of Defense Grant DOD W81XWH-15-1-0574, NIH Grants U19 Al67798, U19 Al067773, R01 HL122393, T32 OD010957, and P30CA012197, and the North Carolina Biotechnology Center Grant 2015-IDG-1006. Also supported in part by the National Institutes of Health (R01AG18915; R01AG045551, P30AG021332 and U24AG059624; and The Kermit Glenn Phillips II Chair in Cardiovascular Medicine, Wake Forest School of Medicine (Dr. Kitzman). 


\section{DISCLOSURES}

- Dr. Kitzman has served as a consultant for Bayer, CinRx, Novartis, Relypsa, Abbvie,

GlaxoSmithKline, AstraZeneca, Merck, St. Luke's Medical Center, Duke Clinical Research

Institute, and Corvia Medical; and has received grant funding from Novartis, Bayer,

AstraZeneca, and St. Luke's Medical Center; and holds stock in Gilead Sciences

- Dr. Register has served as a consultant for Novartis, Merck, OHSU, and has received grant

funding from Merck, Organon, and LipimetiX. 


\section{TABLES}

Table 1: Animal Demographics of Previously Irradiated Long Term, Short Term, and Deceased Male Rhesus Macaque Survivors (LT IRR, ST IRR, Deceased IRR) and Controls (LT CTL, ST CTL, Deceased CTL)

\begin{tabular}{|c|c|c|c|c|c|c|c|c|c|}
\hline & $\begin{array}{l}\text { LT IRR } \\
(\mathrm{N}=14)\end{array}$ & $\begin{array}{l}\text { LT CTL } \\
(\mathrm{N}=15)\end{array}$ & $\mathbf{P}$ & $\begin{array}{l}\text { ST IRR } \\
(\mathrm{N}=20)\end{array}$ & $\begin{array}{l}\text { ST CTL } \\
(\mathrm{N}=10)\end{array}$ & $\mathbf{P}$ & $\begin{array}{l}\text { Deceased } \\
\text { IRR }(\mathrm{N}=17)\end{array}$ & $\begin{array}{l}\text { Deceased } \\
\text { CTL }(\mathrm{N}=12)\end{array}$ & $\mathbf{P}$ \\
\hline Age (Years) & $14.96(0.63)$ & $14.15(0.28)$ & 0.25 & $5.47(0.08)$ & $5.25(0.33)$ & 0.40 & $12.14(0.96)$ & $9.33(0.92)$ & 0.47 \\
\hline $\begin{array}{l}\text { Time Since } \\
\text { Irradiation } \\
\text { (Years) }\end{array}$ & $10.48(0.18)$ & --- & --- & $2.51(0.07)$ & --- & --- & $5.80(0.55)$ & --- & --- \\
\hline Dose (Gy) & $7.40(0.16)$ & --- & --- & $7.01(0.14)$ & --- & --- & $6.93(0.13)$ & --- & --- \\
\hline $\begin{array}{c}\text { Body Weight } \\
(\mathrm{kg})\end{array}$ & $11.31(1.11)$ & $14.62(1.25)$ & 0.06 & $9.22(0.56)$ & $8.77(0.35)$ & 0.86 & $13.25(0.85)$ & $10.90(0.54)$ & $0.04^{a}$ \\
\hline $\begin{array}{c}\text { Body Surface } \\
\text { Area }\left(\mathrm{m}^{2}\right)\end{array}$ & $0.48(0.03)$ & $0.55(0.03)$ & 0.16 & $0.42(0.29)$ & $0.41(0.01)$ & 0.97 & $0.54(0.02)$ & $0.47(0.02)$ & 0.06 \\
\hline
\end{tabular}

Data presented as mean (SEM) 


\section{FIGURES}

A

Transmitral E velocity

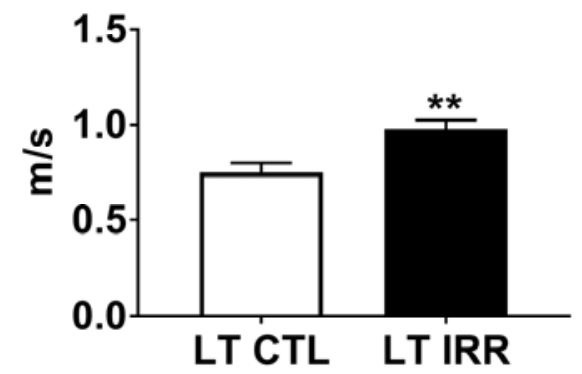

C

$$
\text { E/e' Ratio }
$$

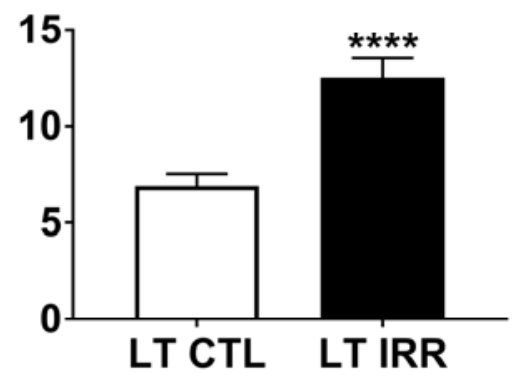

B

Early Mitral Annular Descent Velocity (e')

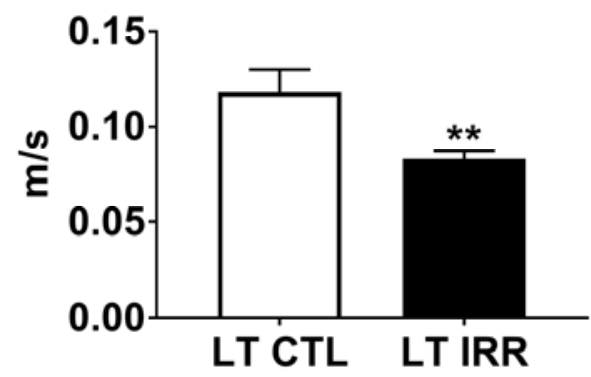

D

\section{Early to Late Mitral Annular Descent Velocity Ratio (e'/a')}

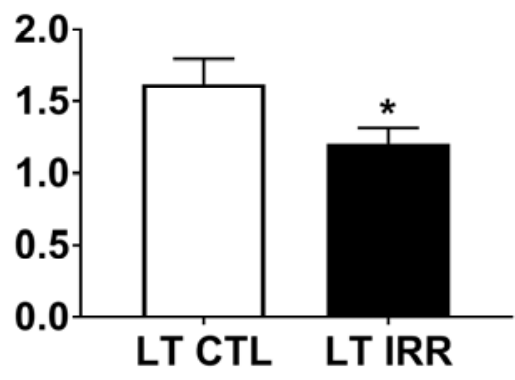

Figure 1 Echocardiographic Diastolic Function in Long Term Irradiated (LT IRR) and Long Term Controls (LT CTL): LT IRR animals had significantly increased transmitral early filling peak velocity (E) (Panel A), decreased early mitral annular descent velocity (e')(Panel B), increased E/e' ratio (Panel C) and decreased early to late mitral annular descent velocity ratio $\left(e^{\prime} / a^{\prime}\right)\left(\right.$ Panel D) compared to LT CTL animals. Data are mean \pm SEM. ${ }^{*} p<0.05,{ }^{* *} p<0.01$, ${ }^{* * * *} p<0.0001$ 
A

Cardiac Output (BP)

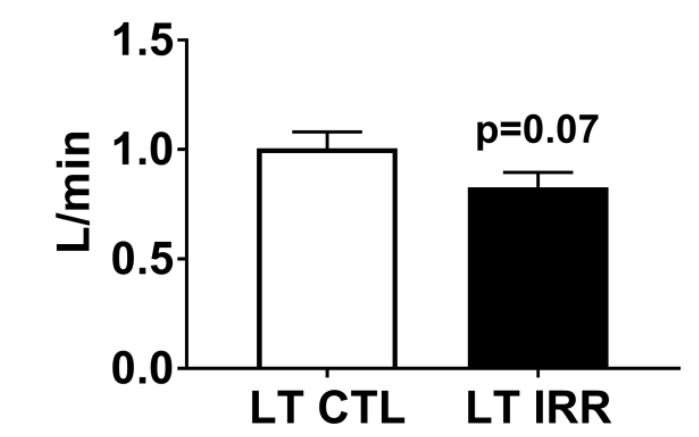

C

Stroke Volume (BP)

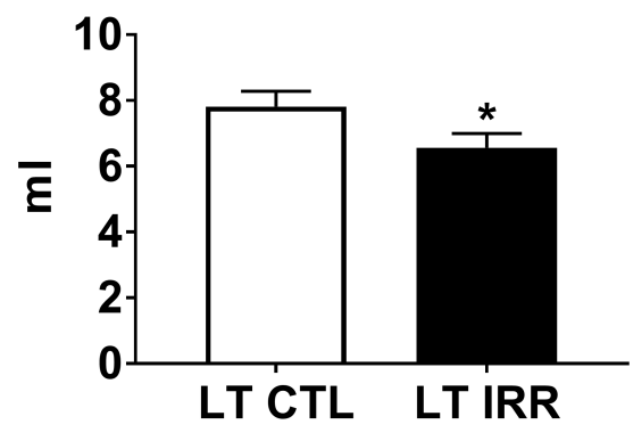

B

\section{Cardiac Output (A4C)}

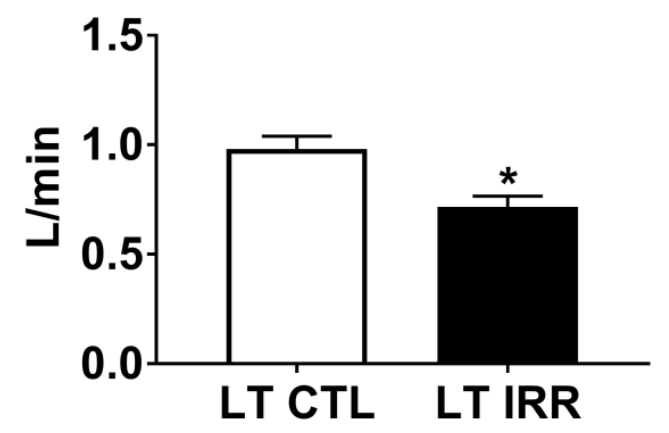

D

End Diastolic Volume (BP)

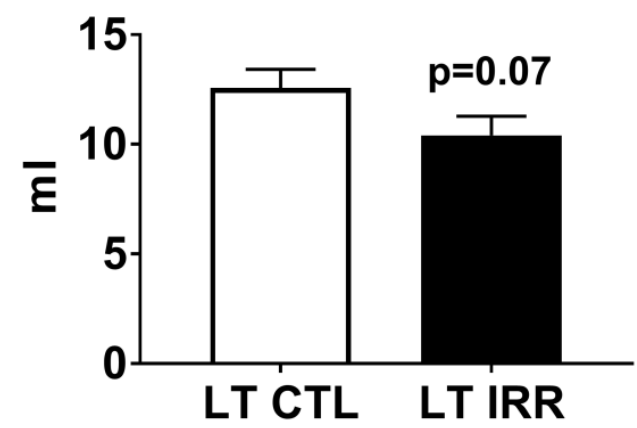

Figure 2 Echocardiographic Cardiac Volume Measures in Long Term Irradiated (LT IRR) and Long Term Controls (LT CTL): LT IRR animals tended to have decreased cardiac output calculated by method of disks measurements using the perpendicularly oriented apical 2 chamber (A2C) and apical 4 chamber (A4C) views resulting biplane measurement (BP) (Panel A). LT IRR animals did have significantly lower cardiac output when only the A4C chamber view was used (Panel B). Additionally, LT IRR animals had decreased stroke volume (BP measurement) and a tendency for decreased end diastolic volume (BP measurement). Data are mean \pm SEM. ${ }^{*} \mathrm{p}<0.05$ 
A

Transmitral E velocity

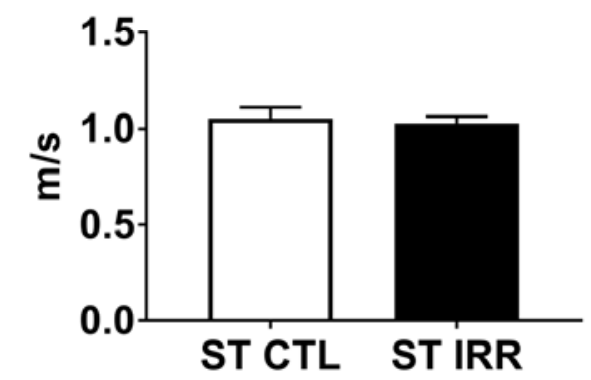

C

$$
\text { E/e' Ratio }
$$

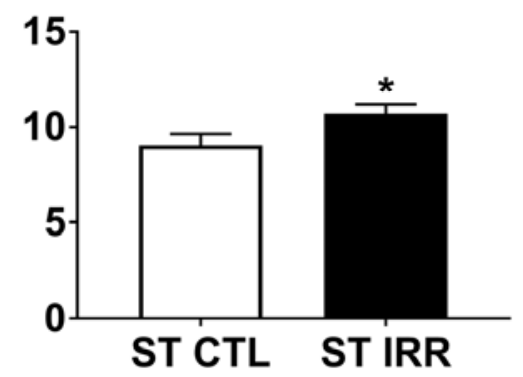

B

Early Mitral Annular Descent Velocity (e')

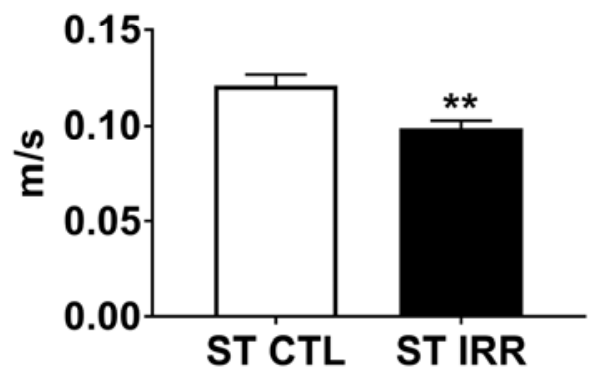

D

\section{Early to Late Mitral Annular Descent Velocity Ratio (e'/a')}

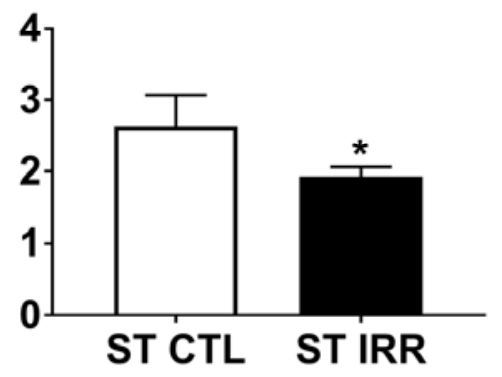

Figure 3 Echocardiographic Diastolic Function in Short Term Irradiated (ST IRR) and Short Term Controls (ST CTL): ST IRR animals had no differences in transmitral early filling peak velocity (E) (Panel A), but decreased early mitral annular descent velocity (e')(Panel B), increased E/e' ratio (Panel C) and decreased early to late mitral annular descent velocity ratio $\left(e^{\prime} / a^{\prime}\right)\left(\right.$ Panel D) compared to ST CTL animals. Data are mean \pm SEM. ${ }^{*} p<0.05,{ }^{* *} p<0.01$ 
A

\section{Trichrome Positive Staining}
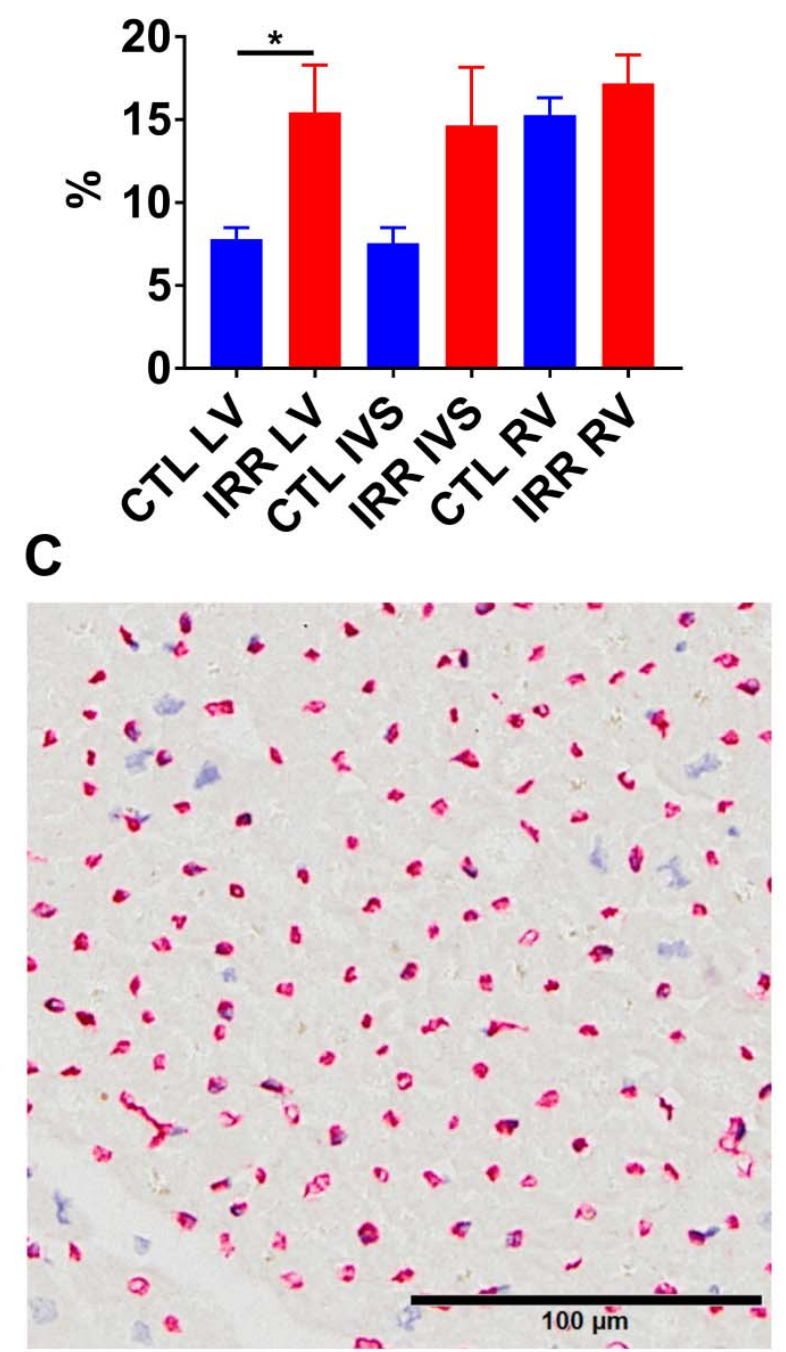

B

\section{CD31 Positive Staining}

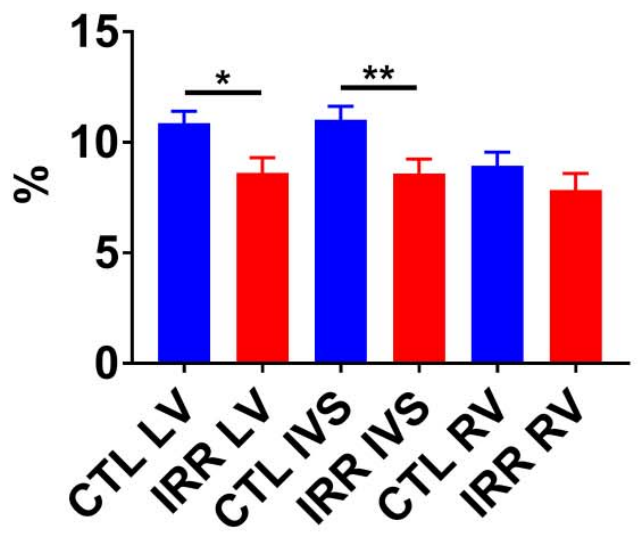

D

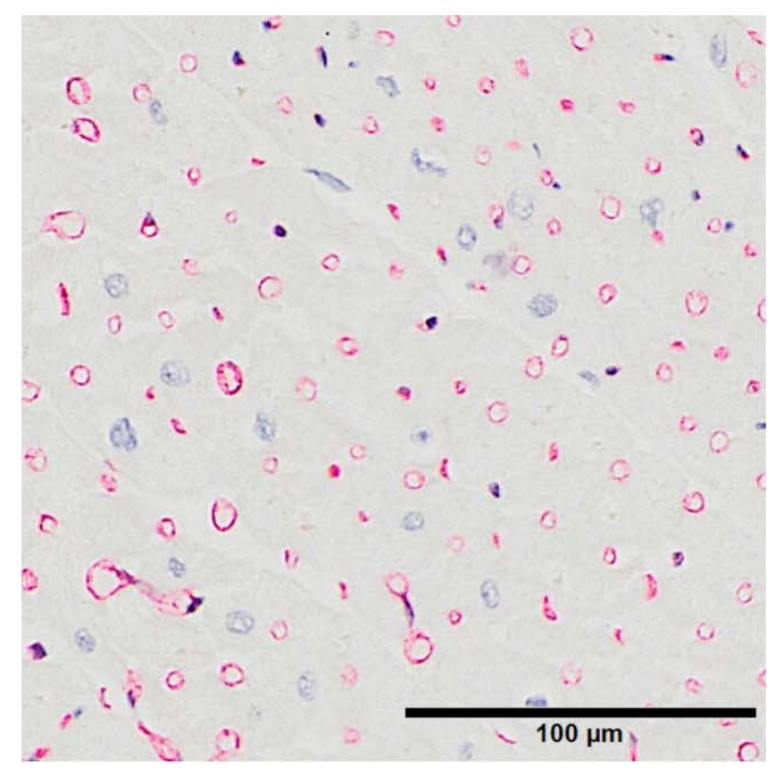

Figure 4 Fibrosis and Capillary Density Immunostaining in Deceased Irradiated (IRR) and

Control (CTL) Animals: IRR animals had significantly increased percent positive trichrome staining of the left ventricle (LV) but not the interventricular septum (IVS) or right ventricle (RV) compared to CTL. IRR animals also had significantly decreased CD31 percent positive staining in the LV and IVS, but not RV compared to CTL (Panel B). Panels C and D are representative CD31 stained sections of LV mid-myocardium from a CTL and IRR animal respectively, demonstrating increased CD31 staining in CTLs. ${ }^{*} p<0.05,{ }^{* *} p<0.01$ 
Figure 5 Fibroblast Immunostaining in

\section{Deceased Irradiated (IRR) and Control (CTL)}

Animals: IRR animals had greater S100A4 staining

in all evaluated sections of myocardium compared

to CTL animals (Panel A). Panels $B$ and $C$ are

representative mid-myocardial S100A4 stained

sections from a CTL and IRR animal respectively

illustrating typical positive staining increases in IRR

animals. ${ }^{*} \mathrm{p}<0.05,{ }^{* *} \mathrm{p}<0.01,{ }^{* * *} \mathrm{p}<0.001$

\section{A}

\section{S100A4 Positive Area}

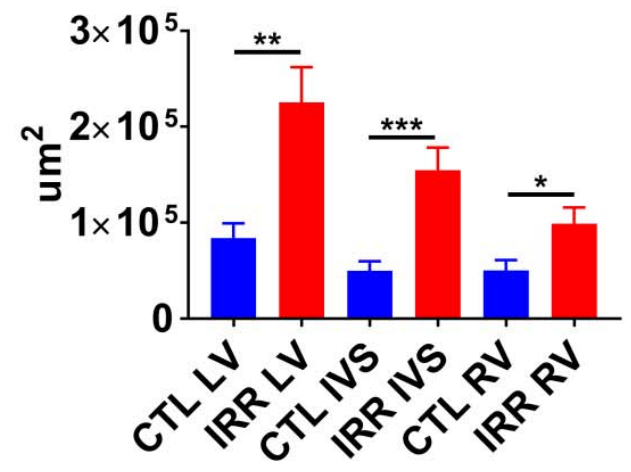

B

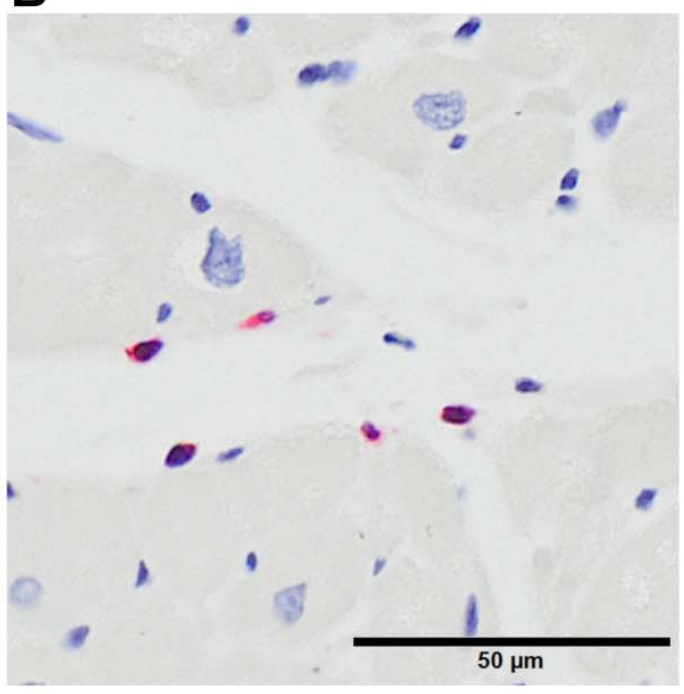

C

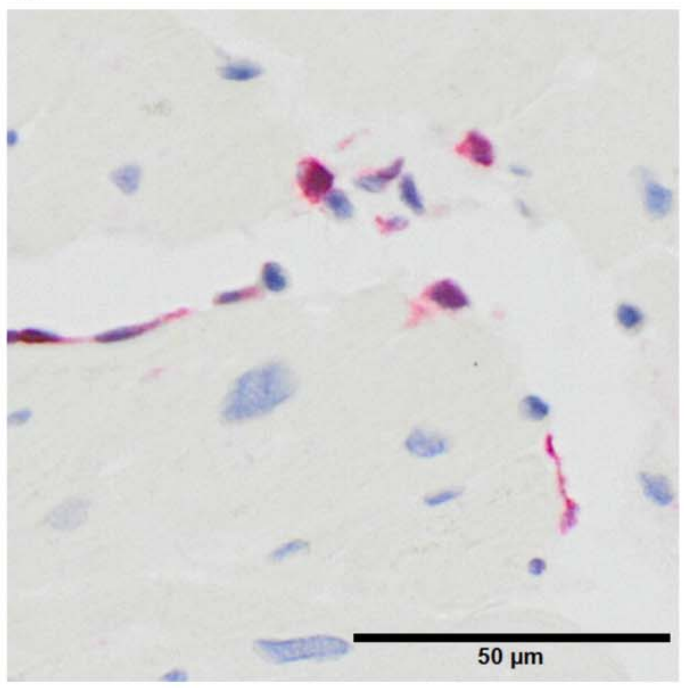


A

\section{CD163 Positive Area}
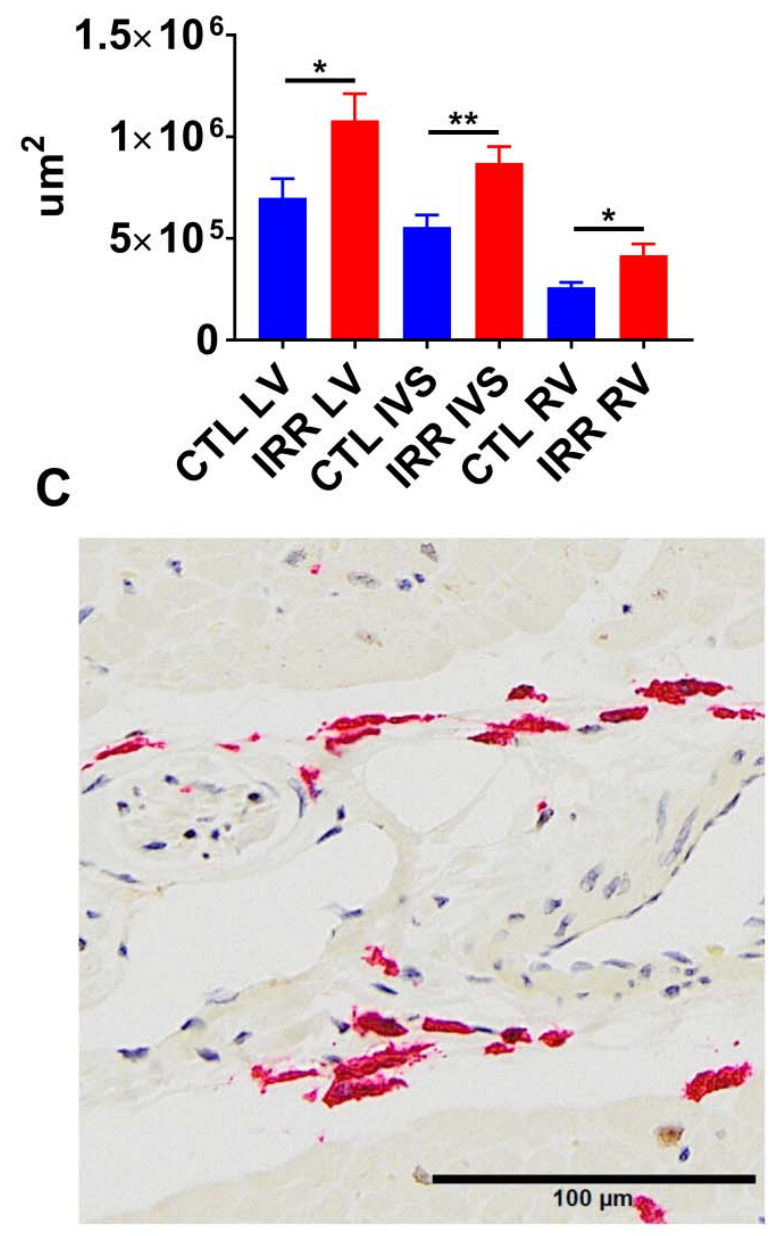

B

\section{CD68 Positive Area}
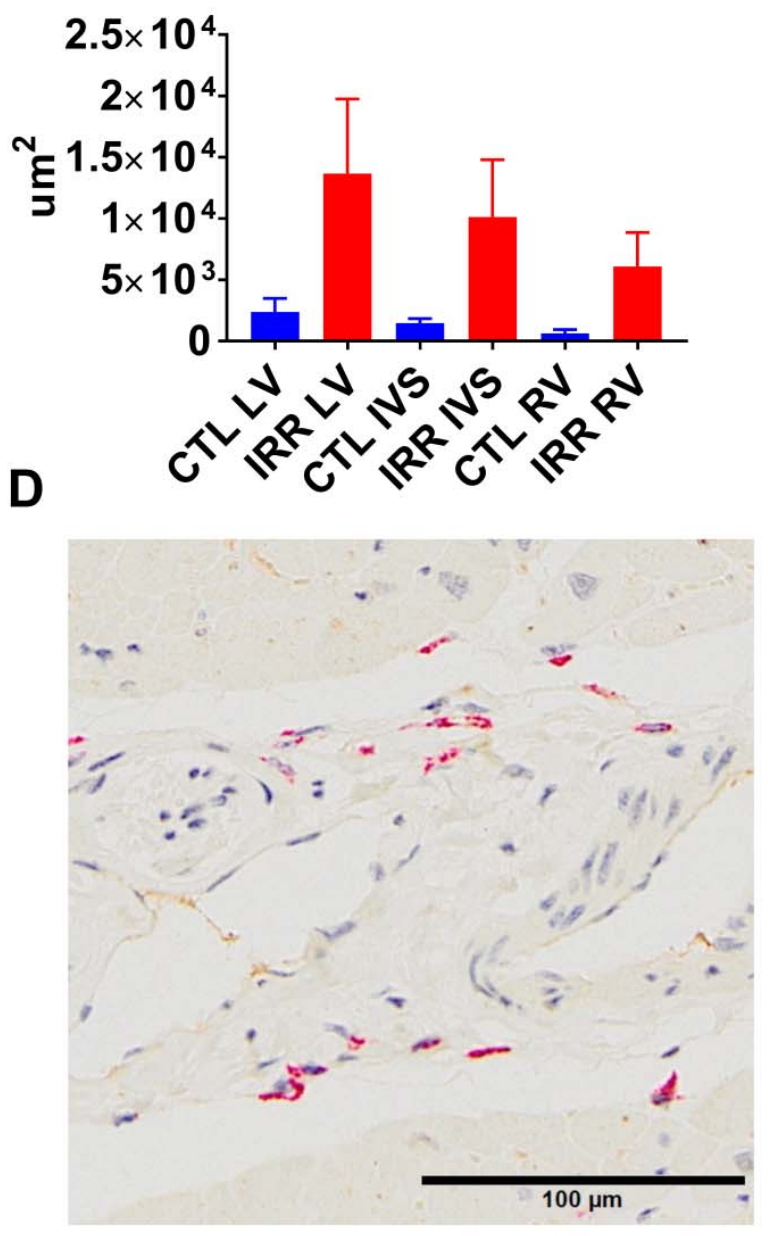

Figure 6 Macrophage Immunostaining in Deceased Irradiated (IRR) and Control (CTL)

Animals: IRR animals had increased normalized CD163 positive staining area in the left ventricle (LV), interventricular septum (IVS), and right ventricle (RV) compared to controls (CTL) (Panel A). In contrast no differences were observed in CD68 staining which was highly variable across both groups (Panel B). Panels $\mathrm{C}$ and D are CD163 and CD68 stains respectively from the same animal in the same location illustrating that $\mathrm{CD} 68^{+}$macrophages were also often CD163 ${ }^{+} .{ }^{*} p<0.05,{ }^{* *} p<0.01$ 
A

c-Maf/CD163 Positive Area (M2)
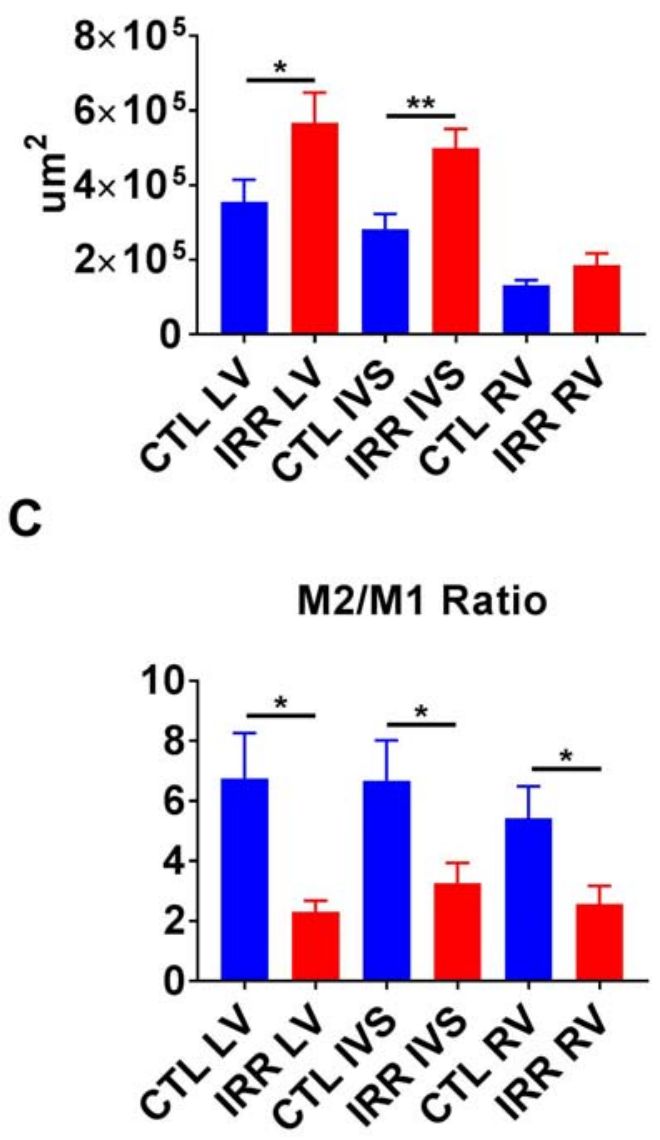

B

pSTAT1/CD163 Positive Area (M1)

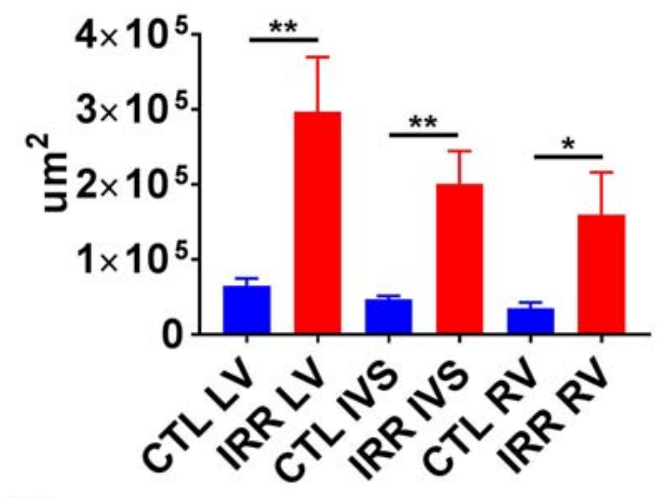

D

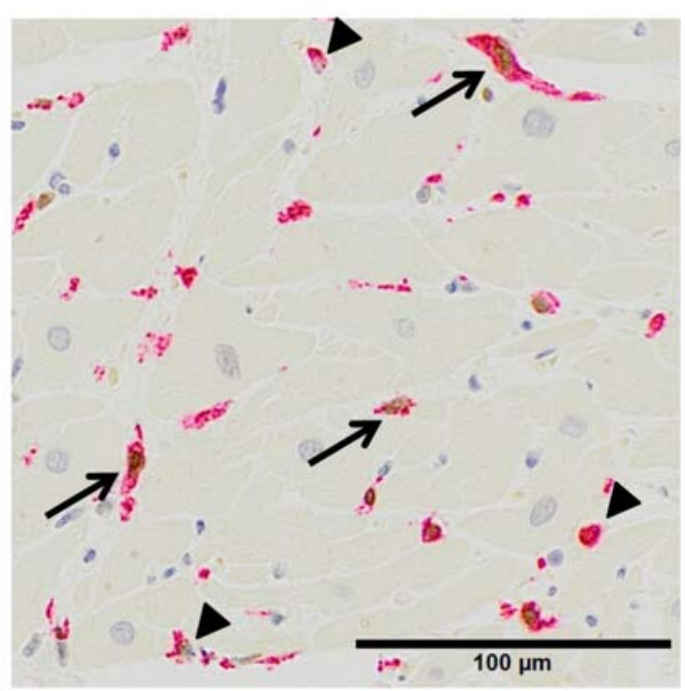

Figure 7 Macrophage Polarization in Irradiated (IRR) and Control (CTL) Myocardium: IRR animals had increased c-Maf ${ }^{+} / \mathrm{CD}_{163^{+}}$staining (M2 polarization) in the left ventricle (LV), interventricular septum (IVS), but not right ventricle (RV) compared to controls (CTL) (Panel A). Increased pSTAT1 $1^{+} / \mathrm{CD} 163^{+}$staining (M1 polarization) was noted in all sections (LV, IVS, and $R V$ ) in IRR animals compared to CTL (Panel B). This translated to a decrease in the M2/M1 macrophage ratio across all sections in IRR animals (Panel C). Panel $D$ is a representative dual stained image indicating dual stained c-Maf $/ \mathrm{CD}^{+} 63^{+}$macrophages (arrows) and single stained $\mathrm{CD}_{163}{ }^{+}$macrophages (arrowheads) from the LV of an irradiated animal. ${ }^{*} \mathrm{p}<0.05,{ }^{* *} \mathrm{p}<0.01$ 


\section{REFERENCES}

1. Stewart JR, Fajardo LF, Gillette SM and Constine LS. Radiation injury to the heart. International journal of radiation oncology, biology, physics. 1995;31:1205-11.

2. Taunk NK, Haffty BG, Kostis JB and Goyal S. Radiation-Induced Heart Disease: Pathologic Abnormalities and Putative Mechanisms. Frontiers in Oncology. 2015;5:39.

3. Fajardo LF, Stewart JR and Cohn KE. Morphology of radiation-induced heart disease. Archives of pathology. 1968;86:512-9.

4. Chello M, Mastroroberto P, Romano R, Zofrea S, Bevacqua I and Marchese AR. Changes in the proportion of types I and III collagen in the left ventricular wall of patients with post-irradiative pericarditis. Cardiovascular Surgery. 1996;4:222-226.

5. Walaszczyk A, Szoltysek K, Jelonek K, Polanska J, Dorr W, Haagen J, Widlak P and Gabrys D. Heart irradiation reduces microvascular density and accumulation of HSPA1 in mice. Strahlenther Onkol. 2018;194:235-242.

6. Patties I, Haagen J, Dorr W, Hildebrandt G and Glasow A. Late inflammatory and thrombotic changes in irradiated hearts of C57BL/6 wild-type and atherosclerosis-prone ApoEdeficient mice. Strahlenther Onkol. 2015;191:172-9.

7. Fajardo LF and Stewart JR. Capillary injury preceding radiation-induced myocardial fibrosis. Radiology. 1971;101:429-33.

8. Douple EB, Mabuchi K, Cullings HM, Preston DL, Kodama K, Shimizu Y, Fujiwara S and Shore RE. Long-term Radiation-Related Health Effects in a Unique Human Population: Lessons Learned from the Atomic Bomb Survivors of Hiroshima and Nagasaki. Disaster medicine and public health preparedness. 2011;5:S122-S133.

9. Yamada M, Wong FL, Fujiwara S, Akahoshi M and Suzuki G. Noncancer disease incidence in atomic bomb survivors, 1958-1998. Radiation research. 2004;161:622-32. 
10. Ozasa K, Shimizu Y, Suyama A, Kasagi F, Soda M, Grant EJ, Sakata R, Sugiyama H and Kodama K. Studies of the mortality of atomic bomb survivors, Report 14, 1950-2003: an overview of cancer and noncancer diseases. Radiation research. 2012;177:229-43.

11. Shimizu Y, Kodama K, Nishi N, Kasagi F, Suyama A, Soda M, Grant EJ, Sugiyama H, Sakata R, Moriwaki H, Hayashi M, Konda M and Shore RE. Radiation exposure and circulatory disease risk: Hiroshima and Nagasaki atomic bomb survivor data, 1950-2003. Bmj. 2010;340:b5349.

12. Takahashi I, Abbott RD, Ohshita T, Takahashi T, Ozasa K, Akahoshi M, Fujiwara S, Kodama K and Matsumoto M. A prospective follow-up study of the association of radiation exposure with fatal and non-fatal stroke among atomic bomb survivors in Hiroshima and Nagasaki (1980-2003). BMJ open. 2012;2:e000654.

13. Adams MJ, Grant EJ, Kodama K, Shimizu Y, Kasagi F, Suyama A, Sakata R and Akahoshi M. Radiation dose associated with renal failure mortality: a potential pathway to partially explain increased cardiovascular disease mortality observed after whole-body irradiation. Radiation research. 2012;177:220-8.

14. Sera N, Hida A, Imaizumi M, Nakashima E and Akahoshi M. The association between chronic kidney disease and cardiovascular disease risk factors in atomic bomb survivors. Radiation research. 2013;179:46-52.

15. Pham TM, Sakata R, Grant EJ, Shimizu Y, Furukawa K, Takahashi I, Sugiyama H, Kasagi F, Soda M, Suyama A, Shore RE and Ozasa K. Radiation exposure and the risk of mortality from noncancer respiratory diseases in the life span study, 1950-2005. Radiation research. 2013;180:539-45.

16. Stewart JR and Fajardo LF. Radiation-induced heart disease. Clinical and experimental aspects. Radiologic clinics of North America. 1971;9:511-31.

17. Lauk S. Endothelial alkaline phosphatase activity loss as an early stage in the development of radiation-induced heart disease in rats. Radiation research. 1987;110:118-28. 
18. Van Der Meeren A, Squiban C, Gourmelon P, Lafont H and Gaugler MH. Differential regulation by IL-4 and IL-10 of radiation-induced IL-6 and IL-8 production and ICAM-1 expression by human endothelial cells. Cytokine. 1999;11:831-8.

19. Gallo RL, Dorschner RA, Takashima S, Klagsbrun M, Eriksson E and Bernfield M. Endothelial cell surface alkaline phosphatase activity is induced by IL-6 released during wound repair. The Journal of investigative dermatology. 1997;109:597-603.

20. Bryan N, Ahswin H, Smart N, Bayon Y, Wohlert S and Hunt JA. Reactive oxygen species (ROS)--a family of fate deciding molecules pivotal in constructive inflammation and wound healing. Eur Cell Mater. 2012;24:249-65.

21. Wermuth PJ and Jimenez SA. The significance of macrophage polarization subtypes for animal models of tissue fibrosis and human fibrotic diseases. Clin Trans/ Med. 2015;4:2.

22. Stewart JR and Fajardo LF. Radiation-induced heart disease: an update. Progress in cardiovascular diseases. 1984;27:173-94.

23. DeBo RJ, Lees CJ, Dugan GO, Caudell DL, Michalson KT, Hanbury DB, Kavanagh K, Cline JM and Register TC. Late Effects of Total-Body Gamma Irradiation on Cardiac Structure and Function in Male Rhesus Macaques. Radiat Res. 2016;186:55-64.

24. Westbury CB and Yarnold JR. Radiation Fibrosis - Current Clinical and Therapeutic Perspectives. Clinical Oncology. 2012;24:657-672.

25. Michalson KT, Macintyre AN, Sempowski GD, Bourland JD, Howard TD, Hawkins GA, Dugan GO, Cline JM and Register TC. Monocyte Polarization is Altered by Total-Body Irradiation in Male Rhesus Macaques: Implications for Delayed Effects of Acute Radiation Exposure. Radiation research. 2019;192:121-134.

26. McEniery PT, Dorosti K, Schiavone WA, Pedrick TJ and Sheldon WC. Clinical and angiographic features of coronary artery disease after chest irradiation. The American journal of cardiology. 1987;60:1020-4. 
27. Vasan RS, Larson MG, Benjamin EJ, Evans JC and Levy D. Left Ventricular Dilatation and the Risk of Congestive Heart Failure in People without Myocardial Infarction. New England Journal of Medicine. 1997;336:1350-1355.

28. Heidenreich PA, Hancock SL, Vagelos RH, Lee BK and Schnittger I. Diastolic dysfunction after mediastinal irradiation. American heart journal. 2005;150:977-982.

29. Armstrong GT, Joshi VM, Ness KK, Marwick TH, Zhang N, Srivastava D, Griffin BP, Grimm RA, Thomas J, Phelan D, Collier P, Krull KR, Mulrooney DA, Green DM, Hudson MM, Robison LL and Plana JC. Comprehensive Echocardiographic Detection of Treatment-related Cardiac Dysfunction in Adult Survivors of Childhood Cancer: Results from the St. Jude Lifetime Cohort Study. Journal of the American College of Cardiology. 2015;65:2511-2522.

30. Hanbury DB, Peiffer AM, Dugan G, Andrews RN and Cline JM. Long-Term Cognitive Functioning in Single-Dose Total-Body Gamma-Irradiated Rhesus Monkeys ( Macaca mulatta ). Radiation research. 2016;186:447-454.

31. Fanning KM, Pfisterer B, Davis AT, Presley TD, Williams IM, Wasserman DH, Cline JM and Kavanagh K. Changes in microvascular density differentiate metabolic health outcomes in monkeys with prior radiation exposure and subsequent skeletal muscle ECM remodeling. American journal of physiology Regulatory, integrative and comparative physiology. 2017;313:R290-r297.

32. Kavanagh K, Dendinger MD, Davis AT, Register TC, DeBo R, Dugan G and Cline JM. Type 2 Diabetes is a Delayed Late Effect of Whole-Body Irradiation in Nonhuman Primates. Radiation research. 2015;183:398-406.

33. Yan G, Zhang G, Fang X, Zhang Y, Li C, Ling F, Cooper DN, Li Q, Li Y, van Gool AJ, Du H, Chen J, Chen R, Zhang P, Huang Z, Thompson JR, Meng Y, Bai Y, Wang J, Zhuo M, Wang T, Huang Y, Wei L, Li J, Wang Z, Hu H, Yang P, Le L, Stenson PD, Li B, Liu X, Ball EV, An N, Huang Q, Zhang Y, Fan W, Zhang X, Li Y, Wang W, Katze MG, Su B, Nielsen R, Yang H, Wang J, Wang $X$ and Wang J. Genome sequencing and comparison of two nonhuman primate animal 
models, the cynomolgus and Chinese rhesus macaques. Nature Biotechnology. 2011;29:10191023.

34. Williams JP, Brown SL, Georges GE, Hauer-Jensen M, Hill RP, Huser AK, Kirsch DG, Macvittie TJ, Mason KA, Medhora MM, Moulder JE, Okunieff P, Otterson MF, Robbins ME, Smathers JB and McBride WH. Animal models for medical countermeasures to radiation exposure. Radiation research. 2010;173:557-578.

35. Singh VK, Newman VL, Berg AN and MacVittie TJ. Animal models for acute radiation syndrome drug discovery. Expert Opinion on Drug Discovery. 2015;10:497-517.

36. Singh VK, Newman VL, Berg AN and MacVittie TJ. Animal models for acute radiation syndrome drug discovery. Expert opinion on drug discovery. 2015;10:497-517.

37. Messaoudi I, Estep R, Robinson B and Wong SW. Nonhuman primate models of human immunology. Antioxidants \& redox signaling. 2011;14:261-273.

38. Palermo RE, Tisoncik-Go J, Korth MJ and Katze MG. Old world monkeys and new age science: the evolution of nonhuman primate systems virology. ILAR J. 2013;54:166-180.

39. Augustine AD, Gondre-Lewis T, McBride W, Miller L, Pellmar TC and Rockwell S. Animal models for radiation injury, protection and therapy. Radiation research. 2005;164:100-9. 40. Roome N. Heart Collection Protocol of the Laboratory Dog and Monkey. Toxicologic pathology. 2005;33:738-739.

41. Nagueh SF, Smiseth OA, Appleton CP, Byrd BF, 3rd, Dokainish H, Edvardsen T, Flachskampf FA, Gillebert TC, Klein AL, Lancellotti P, Marino P, Oh JK, Popescu BA and Waggoner AD. Recommendations for the Evaluation of Left Ventricular Diastolic Function by Echocardiography: An Update from the American Society of Echocardiography and the European Association of Cardiovascular Imaging. Journal of the American Society of Echocardiography : official publication of the American Society of Echocardiography. 2016;29:277-314. 
42. Lang RM, Badano LP, Mor-Avi V, Afilalo J, Armstrong A, Ernande L, Flachskampf FA, Foster E, Goldstein SA, Kuznetsova T, Lancellotti P, Muraru D, Picard MH, Rietzschel ER, Rudski L, Spencer KT, Tsang W and Voigt JU. Recommendations for cardiac chamber quantification by echocardiography in adults: an update from the American Society of Echocardiography and the European Association of Cardiovascular Imaging. Eur Heart $J$ Cardiovasc Imaging. 2015;16:233-70.

43. Korcarz CE, Padrid PA, Shroff SG, Weinert L and Lang RM. Doppler echocardiographic reference values for healthy rhesus monkeys under ketamine hydrochloride sedation. Journal of medical primatology. 1997;26:287-98.

44. Park J-H and Marwick TH. Use and Limitations of E/e' to Assess Left Ventricular Filling Pressure by Echocardiography. Journal of Cardiovascular Ultrasound. 2011;19:169-173.

45. Liu CT and Higbee GA. Determination of body surface area in the rhesus monkey. $J$ Appl Physiol. 1976;40:101-4.

46. Barros MHM, Hauck F, Dreyer JH, Kempkes B and Niedobitek G. Macrophage Polarisation: an Immunohistochemical Approach for Identifying M1 and M2 Macrophages. PloS one. 2013;8:e80908.

47. Lo Q and Thomas L. Echocardiographic evaluation of diastolic heart failure. Australasian journal of ultrasound in medicine. 2010;13:14-26.

48. Nagueh SF, Middleton KJ, Kopelen HA, Zoghbi WA and Quiñones MA. Doppler Tissue Imaging: A Noninvasive Technique for Evaluation of Left Ventricular Relaxation and Estimation of Filling Pressures. Journal of the American College of Cardiology. 1997;30:1527-1533.

49. Kasner M, Westermann D, Steendijk P, Gaub R, Wilkenshoff U, Weitmann K, Hoffmann W, Poller W, Schultheiss HP, Pauschinger M and Tschope C. Utility of Doppler echocardiography and tissue Doppler imaging in the estimation of diastolic function in heart failure with normal ejection fraction: a comparative Doppler-conductance catheterization study. Circulation. 2007;116:637-47. 
50. Moreo A, Ambrosio G, De Chiara B, Pu M, Tran T, Mauri F and Raman SV. Influence of myocardial fibrosis on left ventricular diastolic function: noninvasive assessment by cardiac magnetic resonance and echo. Circulation Cardiovascular imaging. 2009;2:437-443.

51. Bocchi EA, Kalil R, Bacal F, de Lourdes Higuchi M, Meneghetti C, Magalhaes A, Belotti G and Ramires JA. Magnetic Resonance Imaging in Chronic Chagas' Disease: Correlation with Endomyocardial Biopsy Findings and Gallium-67 Cardiac Uptake. Echocardiography. 1998;15:279-288.

52. Mahrholdt H, Goedecke C, Wagner A, Meinhardt G, Athanasiadis A, Vogelsberg H, Fritz $\mathrm{P}$, Klingel K, Kandolf $\mathrm{R}$ and Sechtem U. Cardiovascular magnetic resonance assessment of human myocarditis: a comparison to histology and molecular pathology. Circulation. 2004;109:1250-8.

53. Dokainish H, Zoghbi WA, Lakkis NM, Ambriz E, Patel R, Quinones MA and Nagueh SF. Incremental predictive power of B-type natriuretic peptide and tissue Doppler echocardiography in the prognosis of patients with congestive heart failure. Journal of the American College of Cardiology. 2005;45:1223-1226.

54. Yamamoto T, Oki T, Yamada H, Tanaka H, Ishimoto T, Wakatsuki T, Tabata T and Ito S. Prognostic value of the atrial systolic mitral annular motion velocity in patients with left ventricular systolic dysfunction. Journal of the American Society of Echocardiography. 2003;16:333-339.

55. Hillis GS, Møller JE, Pellikka PA, Gersh BJ, Wright RS, Ommen SR, Reeder GS and Oh JK. Noninvasive estimation of left ventricular filling pressure by e/e' is a powerful predictor of survival after acute myocardial infarction. Journal of the American College of Cardiology. 2004;43:360-367.

56. Saiki H, Petersen IA, Scott CG, Bailey KR, Dunlay SM, Finley RR, Ruddy KJ, Yan E and Redfield MM. Risk of Heart Failure With Preserved Ejection Fraction in Older Women After Contemporary Radiotherapy for Breast Cancer. Circulation. 2017;135:1388-1396. 
57. Cao L, Cai G, Chang C, Miao A-Y, Yu X-L, Yang Z-Z, Ma J-L, Zhang Q, Wu J, Guo X-M and Chen J-Y. Diastolic Dysfunction Occurs Early in HER2-Positive Breast Cancer Patients Treated Concurrently With Radiation Therapy and Trastuzumab. The oncologist. 2015;20:605614.

58. Hudson JC, Baum ST, Frye DM, Roecker EB and Kemnitz JW. Age and sex differences in body size and composition during rhesus monkey adulthood. Aging (Milan, Italy). 1996;8:197204.

59. Strutz F, Okada H, Lo CW, Danoff T, Carone RL, Tomaszewski JE and Neilson EG. Identification and characterization of a fibroblast marker: FSP1. J Cell Biol. 1995;130:393-405. 60. Schneider M, Kostin S, Strom CC, Aplin M, Lyngbaek S, Theilade J, Grigorian M, Andersen CB, Lukanidin E, Lerche Hansen J and Sheikh SP. S100A4 is upregulated in injured myocardium and promotes growth and survival of cardiac myocytes. Cardiovascular research. $2007 ; 75: 40-50$

61. Zeisberg EM and Kalluri R. Origins of cardiac fibroblasts. Circulation research. 2010;107:1304-1312.

62. Kong $P$, Christia $P$, Saxena A, Su Y and Frangogiannis NG. Lack of specificity of fibroblast-specific protein 1 in cardiac remodeling and fibrosis. Am J Physiol Heart Circ Physiol. 2013;305:H1363-72.

63. Österreicher $\mathrm{CH}$, Penz-Österreicher M, Grivennikov SI, Guma M, Koltsova EK, Datz C, Sasik R, Hardiman G, Karin M and Brenner DA. Fibroblast-specific protein 1 identifies an inflammatory subpopulation of macrophages in the liver. Proceedings of the National Academy of Sciences of the United States of America. 2011;108:308-313.

64. Wynn TA and Barron L. Macrophages: master regulators of inflammation and fibrosis. Seminars in liver disease. 2010;30:245-257.

65. Nahrendorf M, Swirski FK, Aikawa E, Stangenberg L, Wurdinger T, Figueiredo J-L, Libby $\mathrm{P}$, Weissleder R and Pittet MJ. The healing myocardium sequentially mobilizes two monocyte 
subsets with divergent and complementary functions. The Journal of experimental medicine. 2007;204:3037-3047.

66. Katze MG, He Y and Gale Jr M. Viruses and interferon: a fight for supremacy. Nature Reviews Immunology. 2002;2:675.

67. Cao S, Liu J, Song $L$ and Ma X. The Protooncogene c-Maf Is an Essential Transcription Factor for IL-10 Gene Expression in Macrophages. The Journal of Immunology. 2005;174:34843492.

A

LV Posterior Wall Thickness End Diastole

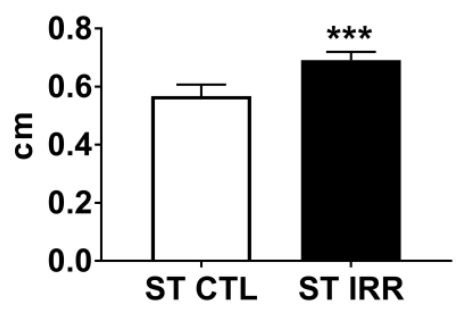

C

Interventricular Septum Thickness End Diastole

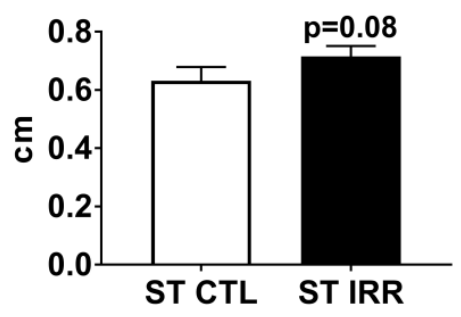

B

LV Posterior Wall Thickness End Systole

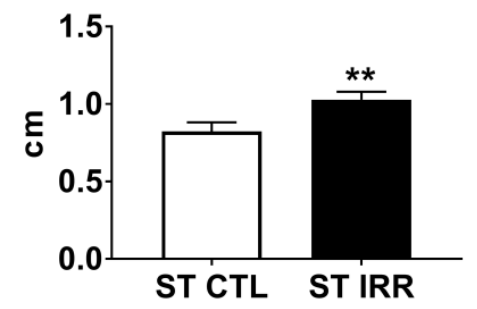

D

Interventricular Septal Thickness End Systole

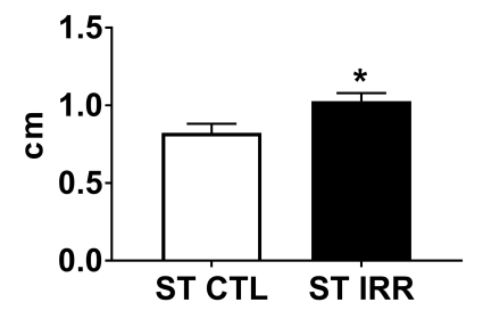

Figure S1 Echocardiographic Structural Difference in Short Term Irradiated (ST IRR) and

Short Term Controls (ST CTL): ST IRR animals had an increased left ventricular posterior wall thickness at both end diastolic and end systole (Panels A \& B), a tendency for increased interventricular septal thickness at end diastole (Panel C), and significantly increased interventricular thickness at end systole (Panel D). Data are mean \pm SEM. ${ }^{*} p<0.05,{ }^{* *} p<0.01$, ${ }^{* * *} \mathrm{p}<0.001$ 